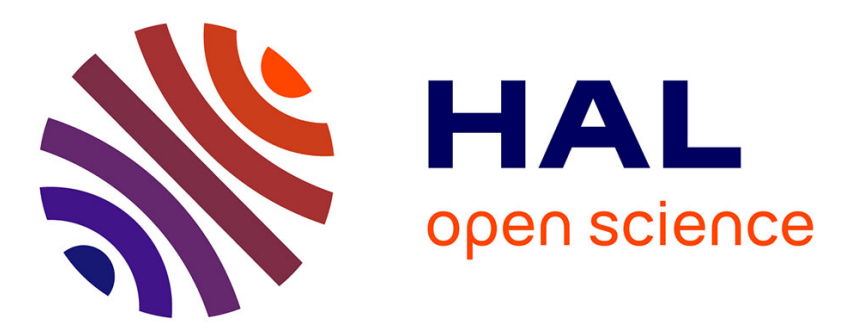

\title{
Efficient Implementation of High Order Reconstruction in Finite Volume Methods
}

Florian Haider, Jean-Pierre Croisille, Bernard Courbet

\section{To cite this version:}

Florian Haider, Jean-Pierre Croisille, Bernard Courbet. Efficient Implementation of High Order Reconstruction in Finite Volume Methods. Finite Volumes for Complex Applications: problems and perspectives, Jun 2011, Prague, Czech Republic. pp.553-562, 10.1007/978-3-642-20671-9_58 . hal01283666

\section{HAL Id: hal-01283666 https://hal.science/hal-01283666}

Submitted on 7 Mar 2016

HAL is a multi-disciplinary open access archive for the deposit and dissemination of scientific research documents, whether they are published or not. The documents may come from teaching and research institutions in France or abroad, or from public or private research centers.
L'archive ouverte pluridisciplinaire HAL, est destinée au dépôt et à la diffusion de documents scientifiques de niveau recherche, publiés ou non, émanant des établissements d'enseignement et de recherche français ou étrangers, des laboratoires publics ou privés. 


\title{
Efficient Implementation of High Order Reconstruction in Finite Volume Methods
}

\author{
Florian Haider $^{1}$, Jean-Pierre Croisille ${ }^{2}$, and Bernard Courbet $^{1}$
}

\section{Introduction}

The finite volume MuscL method to solve hyperbolic conservation laws was introduced by B. Van Leer in $[6,7]$ thirty years ago. The main idea is to increase the accuracy of the first order finite volume scheme by a piecewise linear reconstruction that is used to evaluate upwinded fluxes at the cell interfaces.

Practical applications for convection dominated flows in complex geometries have motivated many extensions of the MUSCL approach to unstructured grids. A typical example is the flow solver CEDRE developed by ONERA. It uses a cell centered finite volume scheme with piecewise linear reconstruction on general polyhedral grids to solve the compressible Navier Stokes equations. A large choice of physical models is available in CEDRE: turbulence (RANS, LES), combustion, diphasic flow, radiation etc.

Our experience has shown that second order accuracy becomes insufficient for LES and to capture contact discontinuities. The easiest way to increase the spatial accuracy is to replace the linear interpolation by quadratic or cubic ones. Indeed, the MuscL scheme with quadratic reconstruction $\left(3^{\text {rd }}\right.$ order) was already discussed by B. Van Leer [6]. The quadratic approach was extended to unstructured grids [1, 2]. The need for large (non compact) stencils seems to have limited the use of cubic reconstructions ( $4^{\text {th }}$ order), although some practical applications exist [5].

For reasons of performance, the computation of a polynomial reconstruction on a grid cell must be local, i.e. make use of data in neighboring cells only. Ideally, the approximation algorithm should only retrieve data from adjacent cells. On the other hand, high order approximation requires a sufficient

ONERA 29 avenue de la Division Leclerc 92322 Châtillon France florian.haider@onera.fr . Université Paul Verlaine-Metz - UFR MIM Département de Mathématiques Ile du Saulcy 57045 Metz Cedex 1 France croisil@poncelet.univ-metz.fr 
number of data samples, which means that data from cells further away in the grid must be accessed.

This paper shows how to compute a high order approximant in a given cell using only data from adjacent cells at a time, eliminating the need to handle directly large reconstruction stencils. No additional degrees of freedom are added: the independent variables are the cell averages of the conserved quantity. The resulting high order finite volume method is well suited for modern parallel and vector (array) computers. This aspect is of primary importance for large scale industrial software.

\section{Semi-discrete High Order Finite Volume Scheme}

- Geometric notation: an unstructured grid is a triangulation of a domain $\Omega \subset \mathbb{R}^{d}$ consisting of $N$ general polyhedra. The cell with number $\alpha$ is denoted $\mathcal{T}_{\alpha}$, with barycenter $\boldsymbol{x}_{\alpha}$ and $d$-volume $\left|\mathcal{T}_{\alpha}\right|$. The face $\mathcal{A}_{\alpha \beta}$, with barycenter $\boldsymbol{x}_{\alpha \beta}$, has a normal vector $\boldsymbol{a}_{\alpha \beta}$ oriented from cell $\mathcal{T}_{\alpha}$ to $\mathcal{T}_{\beta}$ and of length $\left\|\boldsymbol{a}_{\alpha \beta}\right\|$ equal to the surface $\left|\mathcal{A}_{\alpha \beta}\right|$. The oriented normal unit vector of the face $\mathcal{A}_{\alpha \beta}$ is $\boldsymbol{\nu}_{\alpha \beta}$. Furthermore, define $\boldsymbol{h}_{\alpha \beta}=\boldsymbol{x}_{\beta}-\boldsymbol{x}_{\alpha}$. Note that the faces $\mathcal{A}_{\alpha \beta}$ need not be flat, see [4]. One associates with two cells $\mathcal{T}_{\alpha}$ and $\mathcal{T}_{\beta}$ the symmetric tensors

$$
\boldsymbol{z}_{\alpha \beta}^{(k)} \triangleq \frac{1}{\left|\mathcal{T}_{\beta}\right|} \int_{\mathcal{T}_{\beta}}\left(\boldsymbol{x}-\boldsymbol{x}_{\alpha}\right)^{k} d x=\frac{1}{\left|\mathcal{T}_{\beta}\right|} \int_{\mathcal{T}_{\beta}}\left(\boldsymbol{x}-\boldsymbol{x}_{\alpha}\right) \underset{m \text { factors }}{\otimes} \underset{m}{\otimes}\left(\boldsymbol{x}-\boldsymbol{x}_{\alpha}\right) d x
$$

The $k^{\text {th }}$ moment of cell $\mathcal{T}_{\alpha}$ is then defined as $\boldsymbol{x}_{\alpha}^{(k)} \triangleq \boldsymbol{z}_{\alpha \alpha}^{(k)}$. Note that $\boldsymbol{x}_{\alpha}^{(1)}=0$. For a locally integrable function $u$, define its average over cell $\mathcal{T}_{\alpha}$ as $\bar{u}_{\alpha}$.

- Semi-discrete Muscl scheme: consider a hyperbolic conservation law with flux $f$ and write its balance equation over a grid cell $\mathcal{T}_{\alpha}$

$$
\frac{\mathrm{d} \bar{u}_{\alpha}(t)}{\mathrm{d} t}=-\frac{1}{\left|\mathcal{T}_{\alpha}\right|} \sum_{\beta} \int_{\mathcal{A}_{\alpha \beta}} \boldsymbol{\nu}_{\alpha \beta} \cdot \boldsymbol{f}(u(\boldsymbol{x}, t)) d \sigma .
$$

The semi-discrete MuscL discretization of such a conservation law gives the finite volume scheme

$$
\frac{\mathrm{d} \bar{u}_{\alpha}(t)}{\mathrm{d} t}=-\frac{1}{\left|\mathcal{T}_{\alpha}\right|} \sum_{\beta} \int_{\mathcal{A}_{\alpha \beta}} \tilde{f}_{\alpha \beta}\left(w_{\alpha}[\overline{\mathfrak{u}}(t)](\boldsymbol{x}), w_{\beta}[\overline{\mathfrak{u}}(t)](\boldsymbol{x})\right) d \sigma .
$$

In (3), $\tilde{f}_{\alpha \beta}: \mathbb{R} \times \mathbb{R} \rightarrow \mathbb{R}$ is a numerical flux that is consistent with $\boldsymbol{f}:$ $\widetilde{f}_{\alpha \beta}(u, u)=\boldsymbol{\nu}_{\alpha \beta} \cdot \boldsymbol{f}(u)$. The functions $w_{\alpha}$ and $w_{\beta}$ are reconstructed from the cell averages $\overline{\mathfrak{u}}(t)=\left(\bar{u}_{1}(t), \ldots, \bar{u}_{N}(t)\right)$. The dependence of $w_{\alpha}$ on the 
cell averages is denoted by square brackets $w_{\alpha}[\overline{\mathfrak{u}}(t)]$ and the dependence on $\boldsymbol{x}$ by $w_{\alpha}[\overline{\mathfrak{u}}(t)](\boldsymbol{x})$.

- Accuracy: the piecewise reconstruction operates on each cell so that only the cell averages in a certain neighborhood - the reconstruction stencil of cell $\mathcal{T}_{\alpha}$ determine the approximant $w_{\alpha}$. Assume that the reconstruction satisfies for all smooth functions $u$ and uniformly in $\boldsymbol{x} \in \mathcal{T}_{\alpha}$ for all cells $\mathcal{T}_{\alpha}$

$$
\left|w_{\alpha}[\overline{\mathfrak{u}}(t)](\boldsymbol{x})-u(\boldsymbol{x}, t)\right| \leq O\left(h^{k+1}\right) .
$$

Then it is easily verified that (3) is $k^{\text {th }}$ order accurate if $\boldsymbol{f}$ is Lipschitz continuous.

- Conservation: the reconstruction is required to be conservative, i.e. the mean value of the function $w_{\alpha}[\overline{\mathfrak{u}}(t)]$ over the cell $\mathcal{T}_{\alpha}$ must always be $\bar{u}_{\alpha}(t)$.

\section{High Order Polynomial Reconstruction}

This section gives a short overview of $k$-exact reconstruction along the line of [1]. The goal is to reconstruct the $w_{\alpha}$ used in (3) in such a way that they satisfy (4). The focus is on the spatial approximation, therefore the time dependency is dropped to simplify the notation.

Let $\mathbb{P}_{k}\left(\mathbb{R}^{d}\right)$ be the space of polynomials of degree $k$ in $\mathbb{R}^{d}$. In each cell $\mathcal{T}_{\alpha}$, the reconstruction process is represented by linear operator

$$
\mathfrak{R}_{\alpha}: \mathbb{R}^{N} \rightarrow \mathbb{P}_{k}\left(\mathbb{R}^{d}\right) \quad ; \quad \overline{\mathfrak{u}} \longmapsto w_{\alpha}[\overline{\mathfrak{u}}] .
$$

Define a neighborhood of cell $\mathcal{T}_{\alpha}$ as a set of cells $\mathbb{W}_{\alpha} \subset\{1 \ldots, N\}$ such that $\alpha \in \mathbb{W}_{\alpha}$ and associate with $\mathbb{W}_{\alpha}$ a local cell average operator

$$
\mathfrak{P}_{k ; \mathbb{W}_{\alpha}}: \mathbb{P}_{k}\left(\mathbb{R}^{d}\right) \rightarrow \mathbb{R}^{N}
$$

given by $\left(\mathfrak{P}_{k ; \mathbb{W}}(p)\right)_{\beta}=\bar{p}_{\beta}$ if $\beta \in \mathbb{W}_{\alpha}$ and $\left(\mathfrak{P}_{k ; \mathbb{W}}(p)\right)_{\beta}=0$ if $\beta \notin \mathbb{W}_{\alpha}$.

A reconstruction operator $\mathfrak{R}_{\alpha}: \mathbb{R}^{N} \rightarrow \mathbb{P}_{k}\left(\mathbb{R}^{d}\right)$ is called $k$-exact if it is a left inverse of (6)

$$
\mathfrak{R}_{\alpha} \mathfrak{P}_{k ; \mathbb{W}_{\alpha}}=\operatorname{Id}_{\mathbb{P}_{k}\left(\mathbb{R}^{d}\right)} .
$$

It can be shown that, under certain conditions, (7) provides an approximation error (4) that is $O\left(h^{k+1}\right)$ [3].

Note the space of symmetric tensors of rank $m$ in $\mathbb{R}^{d}$ as $\mathcal{S}^{m}\left(\mathbb{R}^{d}\right)$ and define for $\boldsymbol{a}, \boldsymbol{b} \in \mathcal{S}^{m}\left(\mathbb{R}^{d}\right)$ and $\boldsymbol{c} \in \mathbb{R}^{d}$ 


$$
\begin{aligned}
\boldsymbol{a} \bullet \boldsymbol{b} & \triangleq \sum_{i_{1}=1}^{d} \cdots \sum_{i_{m}=1}^{d} a_{i_{1} \cdots i_{m}} b_{i_{1} \cdots i_{m}} \\
(\boldsymbol{a} \cdot \boldsymbol{c})_{j_{1} \cdots j_{m-1}} & \triangleq \sum_{j_{m}=1}^{d} a_{j_{1} \cdots j_{m-1} j_{m}} c_{j_{m}} .
\end{aligned}
$$

Call a function $u k$-exact on $\mathbb{W}_{\alpha}$ if the restriction of $u$ to the cells in $\mathbb{W}_{\alpha}$ is a polynomial of degree $k$ and view the $m^{\text {th }}$ derivative of $u$ as an element of $\mathcal{S}^{m}\left(\mathbb{R}^{d}\right)$.

A $k$-exact $m^{\text {th }}$ derivative on the neighborhood $\mathbb{W}_{\alpha}$ at cell $\mathcal{T}_{\alpha}$ is defined to be a linear map $\boldsymbol{w}_{\alpha}^{(m \mid k)}: \mathbb{R}^{N} \longrightarrow \mathcal{S}^{m}\left(\mathbb{R}^{d}\right)$ such that for all polynomials $p$ of degree $k$

$$
\boldsymbol{w}_{\alpha}^{(m \mid k)}\left[\mathfrak{P}_{k ; \mathbb{W}_{\alpha}}(p)\right]=\left.D^{(m)} p\right|_{\boldsymbol{x}_{\alpha}} .
$$

Since a polynomial is determined by its cell average and its $m^{\text {th }}$ derivatives at a point $\boldsymbol{x}_{\alpha}$, a $k$-exact reconstruction operator is equivalent to a set of $k$ exact $m^{\text {th }}$ derivatives $\boldsymbol{w}_{\alpha}^{(m \mid k)}$ for $1 \leq m \leq k$. The linearity implies that (10) can be written as

$$
\boldsymbol{w}_{\alpha}^{(m \mid k)}[\overline{\mathfrak{u}}]=\sum_{\beta \in \mathbb{W}_{\alpha}} \boldsymbol{w}_{\alpha \beta}^{(m \mid k)} \bar{u}_{\beta}
$$

In (11), the symmetric tensors $\boldsymbol{w}_{\alpha \beta}^{(m \mid k)}$, called the reconstruction coefficients of $\boldsymbol{w}_{\alpha}^{(m \mid k)}$, depend only on the local cell geometry. A complete set of $\boldsymbol{w}_{\alpha \beta}^{(m \mid k)}$ can be computed by applying (10) to a basis of the space $\mathbb{P}_{k}\left(\mathbb{R}^{d}\right)$ and solving the resulting linear system in the least squares sense. The drawback of this method is that its implementation on a computer requires the computation of (11) over large stencils $\mathbb{W}_{\alpha}$.

With the $k$-exact $m^{\text {th }}$ derivatives $(10)$, the general form of the reconstructed polynomial at cell $\mathcal{T}_{\alpha}$ is

$$
w[\overline{\mathfrak{u}}](\boldsymbol{x})=\bar{u}_{\alpha}+\sum_{m=1}^{k} \frac{1}{m !} \boldsymbol{w}_{\alpha}^{(m \mid k)}[\overline{\mathfrak{u}}] \bullet\left[\left(\boldsymbol{x}-\boldsymbol{x}_{\alpha}\right)^{m}-\boldsymbol{x}_{\alpha}^{(m)}\right]
$$

where $\left(\boldsymbol{x}-\boldsymbol{x}_{\alpha}\right)^{m}$ is defined as in (1) and $\boldsymbol{x}_{\alpha}^{(m)} \triangleq \boldsymbol{z}_{\alpha \alpha}^{(m)}$.

When a $k$-exact $m^{\text {th }}$ derivative (11) is applied to a polynomial $p$ of degree $(k+1)$, the reconstruction error can be expressed as

$$
\boldsymbol{w}_{\alpha}^{(m \mid k)}[\overline{\mathfrak{p}}]-\left.D^{(m)} p\right|_{\boldsymbol{x}_{\alpha}}=\frac{1}{(k+1) !} \sum_{\beta \in \mathbb{W}_{\alpha}} \boldsymbol{w}_{\alpha \beta}^{(m \mid k)}\left(\left.\boldsymbol{z}_{\alpha \beta}^{(k+1)} \bullet D^{(k+1)} p\right|_{\boldsymbol{x}_{\alpha}}\right) .
$$

The interest of $(13)$ is that a $(k+1)$-exact $(k+1)^{\text {th }}$ derivative can be used to compute the right hand side of (13) and to subtract it from $\boldsymbol{w}_{\alpha}^{(m \mid k)}$, making $\boldsymbol{w}_{\alpha}^{(m \mid k)}(k+1)$-exact. 
Finally, we introduce the following smoothing technique: let $\mathbb{V}_{\alpha}$ be the set of direct neighbors of cell $\mathcal{T}_{\alpha}$, including $\mathcal{T}_{\alpha}$ itself. Let $\xi_{\alpha}>0$ be such that $\sum_{\beta \in \mathbb{V}_{\alpha}} \xi_{\alpha}=1$. If a set of $k$-exact $k^{\text {th }}$ derivatives $\boldsymbol{w}_{\beta}^{(k \mid k)}$ is known, one can define a new $k$-exact $k^{\text {th }}$ derivative $\widetilde{\boldsymbol{w}}_{\alpha}^{(k \mid k)}$ as a convex combination

$$
\widetilde{\boldsymbol{w}}_{\alpha}^{(k \mid k)}[\overline{\mathfrak{u}}]=\sum_{\beta \in \mathbb{V}_{\alpha}} \xi_{\beta} \boldsymbol{w}_{\beta}^{(k \mid k)}[\overline{\mathfrak{u}}]
$$

The stencil of (14) is larger which increases stability and robustness, see $[4,3]$.

\section{Efficient Algorithms for High Order Reconstruction}

The computation of (11) involves large (non compact) stencils. To avoid this undesirable feature, we try to compute a $(k+1)$-exact $(k+1)^{\text {th }}$ derivative not directly from the cell averages, but from a family of $k$-exact $k^{\text {th }}$ derivatives $\boldsymbol{w}_{\beta}^{(k \mid k)}$ at cells $\mathcal{T}_{\beta}$ for $\beta$ in a small neighborhood $\mathbb{W}_{\alpha}$ of cell $\mathcal{T}_{\alpha}$. This can be done as follows:

Let $\mathbb{W}_{\alpha}$ be a neighborhood of cell $\mathcal{T}_{\alpha}$ and $\boldsymbol{w}_{\beta}^{(k \mid k)}$ be a family of $k$-exact $k^{\text {th }}$ derivatives at cells $\mathcal{T}_{\beta}$ for $\beta \in \mathbb{W}_{\alpha}$. Assume that $\bigcup_{\beta \in \mathbb{W}_{\alpha}} \mathcal{T}_{\beta}$ is path connected where the paths are piecewise $C^{1}$. Let $m_{\alpha} \triangleq\left|\mathbb{W}_{\alpha}\right|-1$ and define the linear operator

$$
\mathfrak{J}_{\mathbb{W}_{\alpha}}^{(k+1)}: \mathcal{S}^{(k+1)}\left(\mathbb{R}^{d}\right) \longrightarrow\left(\mathcal{S}^{(k)}\left(\mathbb{R}^{d}\right)\right)^{m_{\alpha}}
$$

The $i^{\text {th }}$ component of (15) is defined using (1), (8) and (9) as

$$
\begin{aligned}
\left(\mathfrak{J}_{\mathbb{W}_{\alpha}}^{(k+1)}(\boldsymbol{b})\right)_{i} \triangleq \boldsymbol{b} \cdot \boldsymbol{h}_{\alpha \beta_{i}}+\frac{1}{(k+1) !} \sum_{\gamma} \boldsymbol{w}_{\beta_{i} \gamma}^{(k \mid k)}\left(\boldsymbol{z}_{\beta \gamma}^{(k+1)} \bullet \boldsymbol{b}\right)- & \\
& -\frac{1}{(k+1) !} \sum_{\gamma} \boldsymbol{w}_{\alpha \gamma}^{(k \mid k)}\left(\boldsymbol{z}_{\alpha \gamma}^{(k+1)} \bullet \boldsymbol{b}\right) .
\end{aligned}
$$

The operator $\mathfrak{J}_{\mathbb{W}_{\alpha}}^{(k+1)}$ depends on the $\boldsymbol{w}_{\beta}^{(k \mid k)}$ and satisfies

Proposition 1 ( Functional Identity for Reconstruction ). Let $u$ be a function that is $(k+1)$-exact on $\bigcup_{\beta \in \mathbb{W}_{\alpha}} \mathbb{W}_{\beta}^{(k)}$. Then the following identity holds

$$
\begin{aligned}
\mathfrak{J}_{\mathbb{W}_{\alpha}}^{(k+1)}\left(\left.D^{(k+1)} u\right|_{\boldsymbol{x}_{\alpha}}\right)= \\
\quad=\left(\boldsymbol{w}_{\beta_{1}}^{(k \mid k)}[\overline{\mathfrak{u}}]-\boldsymbol{w}_{\alpha}^{(k \mid k)}[\overline{\mathfrak{u}}], \ldots, \boldsymbol{w}_{\beta_{m_{\alpha}}}^{(k \mid k)}[\overline{\mathfrak{u}}]-\boldsymbol{w}_{\alpha}^{(k \mid k)}[\overline{\mathfrak{u}}]\right) .
\end{aligned}
$$

The main result of this section is 
Proposition 2 $\left((k+1)\right.$-exact $(k+1)^{\text {th }}$ derivative $)$. Assume that the operator $\mathfrak{J}_{\mathbb{W}_{\alpha}}^{(k+1)}$ defined in (16) has a left inverse $\mathfrak{D}_{\mathbb{W}_{\alpha}}^{(k+1)}$. Then the following expression defines a $(k+1)$-exact $(k+1)^{\text {th }}$ derivative on the neighborhood $\bigcup_{\beta \in \mathbb{W}_{\alpha}} \mathbb{W}_{\beta}^{(k)}$ :

$$
\begin{aligned}
& \widetilde{\boldsymbol{w}}_{\alpha}^{(k+1 \mid k+1)}[\overline{\mathfrak{u}}] \triangleq \\
& \triangleq \mathfrak{D}_{\mathbb{W}_{\alpha}}^{(k+1)}\left(\boldsymbol{w}_{\beta_{1}}^{(k \mid k)}[\overline{\mathfrak{u}}]-\boldsymbol{w}_{\alpha}^{(k \mid k)}[\overline{\mathfrak{u}}], \ldots, \boldsymbol{w}_{\beta_{m}}^{(k \mid k)}[\overline{\mathfrak{u}}]-\boldsymbol{w}_{\alpha}^{(k \mid k)}[\overline{\mathfrak{u}}]\right)
\end{aligned}
$$

Prop. 2 gives the following algorithm.

\section{Definition 1 ( $k$-exact Coupled Least Squares Algorithm (CLS) ).}

1. Compute a 1-exact $1^{\text {st }}$ derivative directly from the cell averages on a small stencil.

2. Iterate the following step from $m=1$ to $m=k-1$ at each cell:

a. Compute a $(m+1)$-exact $(m+1)^{\text {th }}$ derivative from a $m$-exact $m^{\text {th }}$ derivative, using the Moore Penrose pseudo inverse of (15).

b. On tetrahedral grids, apply $(14)$ to the $(m+1)$-exact $(m+1)^{\text {th }}$ derivative.

3. Use (13) to obtain $k$-exact $m^{\text {th }}$ derivatives for $1 \leq m \leq k-1$.

Remark 1. The smoothing step $2 \mathrm{~b}$ is important on tetrahedral meshes due to stability considerations, see [4].

\section{Numerical Results}

The test case is the linear advection equation with constant velocity $\boldsymbol{c}=$ $\left(\frac{1}{10}, \frac{1}{5}, 1\right)$ on the unit cube with periodic boundaries. The algorithm of Def. 1 has been tested for quadratic $(k=2$, CLS DEG2) and cubic $(k=3$, CLS DEG3) reconstruction with the cell centered finite volume scheme (3). The numerical flux is the classical upwinded flux

$$
\widetilde{f}_{\alpha \beta}\left(u_{\alpha}, u_{\beta}\right) \triangleq\left(\mathbf{c} \cdot \boldsymbol{\nu}_{\alpha \beta}\right)_{+} u_{\alpha}+\left(\mathbf{c} \cdot \boldsymbol{\nu}_{\alpha \beta}\right)_{-} u_{\beta} .
$$

A reconstruction approach using the direct least squares reconstruction mentioned in Sect. 3 serves as comparison, called DLS DEG1, DLS DEG2 and DLS DEG3 for linear, quadratic and cubic reconstruction.

Tables 1,2 and 3 display the convergence rate for the $\ell_{2}$ error at $t=10$ as a function of the average cell diameter $h_{\text {avg }}$ on three different shapes of grids for the initial condition $u_{0}(x, y, z)=\sin (2 \pi x) \sin (2 \pi y) \sin (2 \pi z)$. The column $N$ displays the number of cells. The number $(n)$ in parentheses indicates that the effective size of the stencil is the $n^{\text {th }}$ neighborhood: The first neighborhood 
consists of the cell and its adjacent neighbors, the second neighborhood is the union of the first neighbors of the first neighbors, etc.

Table 1 Grid convergence : series of tetrahedral grids (CLS with smoothing)

\begin{tabular}{|c|c|c|c|c|c|c|}
\hline$h_{\text {avg }}$ & $N$ & CLS DEG2(4) & CLS DEG3(6) & DLS DEG1(2) & DLS DEG2(3) & DLS DEG3(4) \\
\hline 0.042316 & 5928 & & & & & \\
\hline 0.037870 & 8406 & 2.2661 & 4.4984 & 1.9190 & 1.5232 & 4.8690 \\
\hline 0.032909 & 12817 & 2.3654 & 4.4386 & 1.9881 & 1.8080 & 4.8354 \\
\hline 0.027354 & 22493 & 2.7521 & 4.5814 & 2.2780 & 2.3162 & 5.0287 \\
\hline 0.022707 & 39518 & 2.7832 & 4.3773 & 2.3307 & 2.5409 & 4.8292 \\
\hline 0.018133 & 77770 & 2.8736 & 4.2354 & 2.3583 & 2.7250 & 4.7772 \\
\hline 0.013422 & 192972 & 2.9989 & 4.3158 & 2.3962 & 2.9245 & 4.8433 \\
\hline
\end{tabular}

Table 2 Grid convergence : series of cartesian grids (CLS with smoothing)

\begin{tabular}{|c|c|c|c|c|c|c|}
\hline$h_{\text {avg }}$ & $N$ & CLS DEG2(4) & CLS DEG3(6) & DLS DEG1(2) & DLS DEG2(3) & DLS DEG3(4) \\
\hline 0.045455 & 10648 & & & & & \\
\hline 0.035714 & 21952 & 2.4243 & 4.4868 & 1.9355 & 1.9313 & 4.4325 \\
\hline 0.029412 & 39304 & 2.6872 & 4.5062 & 2.1330 & 2.3892 & 4.4078 \\
\hline 0.025000 & 64000 & 2.8119 & 4.4633 & 2.1923 & 2.6240 & 4.3497 \\
\hline 0.021739 & 97336 & 2.8791 & 4.4349 & 2.2001 & 2.7541 & 4.3267 \\
\hline 0.019231 & 140608 & 2.9175 & 4.3359 & 2.1894 & 2.8310 & 4.2191 \\
\hline 0.017241 & 195112 & 2.9406 & 4.3225 & 2.1720 & 2.8779 & 4.2215 \\
\hline
\end{tabular}

Table 3 Grid convergence : series of polyhedral grids (CLS without smoothing)

\begin{tabular}{|c|c|c|c|c|c|c|}
\hline$h_{\text {avg }}$ & $N$ & CLS DEG2(2) & CLS DEG3(3) & DLS DEG1(2) & DLS DEG2(3) & DLS DEG3(4) \\
\hline \multicolumn{7}{|c|}{0.04478413819} \\
\hline 0.041544 & 17933 & 3.1771 & 5.4782 & 1.3943 & 1.0037 & 4.9159 \\
\hline 0.038507 & 22983 & 2.9794 & 4.9878 & 1.4959 & 1.2227 & 4.5057 \\
\hline 0.033027 & 35595 & 2.4432 & 3.3753 & 1.4035 & 1.3847 & 3.9068 \\
\hline 0.029400 & 52487 & 3.3681 & 4.8044 & 2.1503 & 2.2422 & 5.1493 \\
\hline 0.025212 & 80995 & 2.5399 & 2.3076 & 1.6894 & 1.9848 & 4.0666 \\
\hline 0.021547 & 135609 & 3.5112 & 5.4666 & 2.4056 & 2.8601 & 5.3057 \\
\hline
\end{tabular}

Observe that the reconstruction algorithm CLS of Def. 1 gives the desired convergence rates for quadratic $\left(3^{\text {rd }}\right.$ order) and cubic reconstruction $\left(4^{\text {th }}\right.$ order). The rates are comparable to those for the direct method DLS. 


\section{Conclusion}

The algorithm of Def. 1 avoids large reconstruction stencils in implementing high order finite volume schemes (3). It achieves this goal without introducing additional degrees of freedom. The integration of the CLS algorithm in the CEDRE software is an ongoing work. This requires appropriate limiting techniques in order to deal with monotonicity.

Acknowledgements The authors want to thank P. Brenner who is at the origin of an algorithm that is very close to the one of Def. 1 and that has motivated a large part of the present work.

\section{References}

1. Barth, T.J., Frederickson, P.O.: Higher order solution of the Euler equation on unstructured grids using quadratic reconstruction. In: AIAA 90, AIAA-90-0013, pp. 1-12. AIAA, Reno Nevada (1990)

2. Delanaye, M., Essers, J.A.: Quadratic-reconstruction finite volume scheme for compressible flows on unstructured adaptive grids. AIAA Journal 35(4), 631 - 639 (1997)

3. Haider, F.: Discrétisation en maillage non structuré et applications les. Ph.D. thesis, Université Pierre et Marie Curie Paris VI (2009)

4. Haider, F., Croisille, J.P., Courbet, B.: Stability analysis of the cell centered finitevolume MUSCL method on unstructured grids. Numer. Math. 113, 555 - 600 (2009). DOI 10.1007/s00211-009-0242-6

5. Khosla, S., Dionne, P., Lee, M., Smith, C.: Using fourth order spatial integration on unstructured meshes to reduce LES run time. AIAA 2008-782. 46th AIAA Aerospace Sciences Meeting and Exhibit, AIAA (2008)

6. van Leer, B.: Towards the ultimate conservative difference scheme. IV. A new approach to numerical convection. Journal of Computational Physics 23(3), 276 - 299 (1977). DOI DOI:10.1016/0021-9991(77)90095-X. URL http://www.sciencedirect.com/science/article/B6WHY-4DD1MM2-4J/2/ $61 \mathrm{bfce} 9111 \mathrm{ba} 17 \mathrm{f} 514 \mathrm{bbf} 0 \mathrm{fbdb} 2 \mathrm{f} 2 \mathrm{ee} 4$

7. van Leer, B.: Towards the ultimate conservative difference scheme. V. A second-order sequel to Godunov's method. Journal of Computational Physics 32(1), 101 - 136 (1979). DOI DOI:10.1016/0021-9991(79)90145-1. URL http://www. sciencedirect. com/science/article/B6WHY-4DD1N8T-C5/2/9b051d1cfcff715a3d0f4b7b7b0397cc 